\title{
Progress of Xiao Qinglong Decoction in the Treatment of Chronic Obstructive Pulmonary Disease
}

\author{
Yun Li ${ }^{1}$, Aiwu Liang ${ }^{2, *}$, Jinli Chen ${ }^{1}$, Zhijiang Chen ${ }^{1}$ \\ ${ }^{1}$ Guangxi University of Chinese Medicine, Nanning 530001, China \\ ${ }^{2}$ Ruikang Hospital Affiliated to Guangxi University of Chinese Medicine, Nanning 530001, China \\ *Corresponding Author
}

\begin{abstract}
Chronic obstructive pulmonary disease (COPD) is a common disease with high mortality and disability rate of the respiratory system. Xiao Qinglong Decoction has a definite curative effect in treating COPD. This paper will review the research situation and mechanism of Xiao Qinglong Decoction in the treatment of COPD in recent years, providing more evidence for the treatment of COPD with Traditional Chinese medicine.
\end{abstract}

Keywords: Xiao Qinglong Soup, Chronic obstructive pulmonary disease, Experimental research, Clinical research, Reviewed in this paper.

\section{Introduction}

Chronic obstructive pulmonary disease (COPD) is a common preventable and treatable disease characterized by respiratory symptoms (cough, expectoration, dyspnea) and airflow restriction. Chronic obstructive pulmonary disease (COPD) is the fourth leading cause of death globally, and is expected to rise to the third leading cause of death globally after 2020[1]. High rates of COPD reduce patients' quality of life, with dyspnea, limited functional ability, reduced exercise tolerance, impaired health-related quality of life, repeated need for hospitalization, and increased rates of anxiety and depression. The adverse social and economic impact of chronic respiratory diseases on individuals, families, society is significant and is expected to increase significantly in the future[2]. The Global Initiative for Chronic Obstructive Pulmonary Disease (GOLD) guidelines recommend comprehensive management that includes both drug and non-drug therapy and is beneficial for patients with COPD who alleviate symptoms and reduce the frequency of acute exacerbations. Some COPD patients experience difficulty breathing and reduced quality of life despite receiving several treatments recommended in the GOLD guidelines). Therefore, COPD is a priority problem to be solved in our work[3].

\section{Etiology}

Smoking is the main cause of COPD, and genetic factors, age, and gender, lung growth and development, socioeconomic status, asthma, chronic bronchitis, infection, other factors are also affecting the incidence or deterioration of COPD. In the 2022 GOLD Guidelines, it is pointed out that recurrent lower respiratory tract infections, family history of COPD, low birth weight, other factors are key indicators for the diagnosis of COPD, and attention should also be paid to the etiology of disease diagnosis[1]. At present, the western medicine treatment of COPD is mainly the use of bronchodilators, glucocorticoids, antibiotics, other drugs, although COPD patients have received treatment the effect is still not ideal, many adverse reactions of long-term drug use is also a big problem. Therefore, it is of great significance to seek other effective treatments[4].
And without COPD disease of TCM, TCM believes that chronic obstructive pulmonary disease belongs to the category of lung distension, disease in the lungs, spleen, kidney can be involvement, The lung is the root of the disease resistance of phlegm turbidity, think lung expansion for the pathogenesis of exogenous cold evil, and lung Xuan Su, kidney Yang deficiency, lung spleen kidney disorders caused by the liquid water distribution company, stop drinking lung[5]. And Xiao Qinglong soup from the Han Dynasty Zhang Zhongjing "treatise on typhoid", "typhoid table does not understand, the heart has moisture,.......Less full belly or asthma, Xiao Qinglong soup Lord." Xiao Qinglong decoction is composed of ephedra, peony, cassia twig, Schisandra fruit, dried ginger, Pinellia ternata, Asarum, and prepared glycyrrhiza. Studies have proved that Xiao Qinglong Decoction can be widely used for COPD of patients with external cold and internal drink, phlegm (dampness), and latent lung, which can be characterized by cough, expectoration, expectoration salivation or foam-like sputum, wheezing, shortness of breath, chest tightness and shortness of breath, or fever, chills and poor urination, with white tongue coating, smooth pulse or tight floating[6]. Modern medical research has confirmed that Xiao Qinglong Decoction has a good effect on reducing the level of serum inflammatory factors in patients, improving the immune function of patients, and airway remodeling. At present, it has been widely used in the clinical treatment of various respiratory diseases and other clinical diseases.

\section{Experimental Study on Xiao Qinglong Soup}

\subsection{Inhibition of Malondialdehyde (MDA)}

Inhibition of malondialdehyde (MDA) is the main metabolite in the oxidative damage process of the cell membrane, which can lead to cell membrane damage, degeneration, and necrosis. Its content can reflect the level of oxygen free group and the degree of peroxidation damage. Zhao Ye et al.[7] studied the effects of Asarum and Xiao Qinglong Decoction on the levels of AChE, SOD, and MDA in the medulla oblongata of rats at cold asthma, and the experimental results showed that free radicals attacked the medulla oblongata tissue during modeling and damaged the cell membrane due to peroxidation, 
thus reducing the antioxidant capacity. However, Asarum and its composition of Xiao Qinglong Decoction can inhibit and repair the peroxidation damage of rats in different degrees in the process of modeling.

\subsection{The Immune Regulation}

After infection with pathogens in COPD patients, alveolar macrophages, as the body's immune cells, coordinate the enlargement of inflammatory response in the airway, damage lung tissue, and aggravate the disease[8]. P13K/AKT and Notch signal transduction pathways can jointly participate in the inflammatory response process in the form of crosslinking and positive feedback, further aggravating lung tissue injury[9]. Zheng Shuang et al.[10] studied the effect of Xiao Qinglong Decoction on the mRNA expressions of PI3K, Akt, and mTOR in the rat model with Xiao Qinglong Decoction syndrome based on the PI3K/Akt/mTOR signaling pathway, and the results showed that the mechanism of Xiao Qinglong Decoction was related to energy metabolism, immune regulation, and inflammatory response reduction. The occurrence and development of Xiao Qinglong Decoction syndrome are related to the up-regulation of PI3K, Akt, and M TOR M RNA, and its therapeutic mechanism is related to the down-regulation of the relative expression levels of PI3K, Akt, and M TOR M RNA.

\subsection{Airway Remodeling}

Airway remodeling is caused by COPD due to chronic inflammation of the airway, and certain damage to the airway tissue and changes in airway wall structure can cause airway remodeling, aggravate airway stenosis, and cause airway obstruction[11]. TGF- $\beta 1$, as a profibrotic cytokine, often shows high expression in pulmonary diseases. It can induce goblet cell and fibroblast proliferation, promote collagen synthesis and secretion, and accelerate the process of airway remodeling. Smad is one of the downstream target molecules of TGF- $\beta 1$, which is involved in the regulation of pulmonary fibrosis. TGF- $\beta 1$ can phosphorylate downstream Smad 2 and $\mathrm{Smad} 3$, and then regulate the transcription of target genes. It has been reported that blocking the TGF- $\beta 1 /$ Smad pathway with inhibitors can effectively reduce the degree of airway remodeling in COPD mice[12]. Yang Xiangzheng et al.[13] found that the mechanism of Xiao Qinglong Decoction plus Jianhe Erchen Decoction in treating CVA rats may lie in up-regulation of GR protein expression, which mediates TGF- $\beta 1 /$ Smad3 signal axis and down-regulates TGF- $\beta 1$ and Smad3 protein expression levels, thereby inhibiting the production of inflammatory cytokines and alleviating the symptoms of CVA disease.

\subsection{Inhibitory Inflammatory Cell}

It was found that $1,25(\mathrm{OH}) 2 \mathrm{D} 3$ plays a complex regulatory role in the immune response of chronic airway inflammation and autoimmune diseases. Peng Jianping et al. Effects of $1,25(\mathrm{OH}) \_2 \mathrm{D} \_3$ on airway inflammation in rats with chronic obstructive pulmonary disease exposed to PM2.5 in vivo It was found that $1,25(\mathrm{OH}) 2 \mathrm{D} 3$ can reduce COPD airway inflammation and injury, and supplementation of 1, $25(\mathrm{OH})$ 2D3 can partially improve airway inflammation in COPD patients with PM2.5 poisoning[14]. Yan Wei et al.[15] studied the effect of Xiao Qinglong decoction on the level of 1,25 dihydroxy vitamin D 3 in bronchial asthma rats with cold Yin Yunfei syndrome, and the results suggested that Xiao Qinglong decoction reduced the serum il-5 content of rats, inhibited the aggregation and infiltration of inflammatory cells, and alleviated airway inflammation. It can effectively increase the level of 1,25 dihydroxy vitamin D 3 in bronchial asthma rats with cold Yin and lung syndrome, thereby improving the activity of various immune cells, enhancing immunity, inhibiting the inflammatory response of bronchial asthma, and improving airway remodeling.

\subsection{Antianaphylaxis}

Thymic stromal lymphopoietin (TSLP) is a newly discovered IL-7-like cytokine that is expressed by skin, gastrointestinal and respiratory epithelial tissues after exposure to foreign allergens and acts on T cells to participate in body immunity. Studies have shown that TSLP plays an important role in the progression of respiratory diseases[16]. Zhang L L et al.[17] studied the intervention effect of Xiao Qinglong Decoction on the expression of IL-4, IL-12, IFN- $\gamma$, and IL-10 in activated dendritic cells (DCs) induced by TSLP. It was found that Xiao Qinglong Decoction could reduce the expression of IL-4 in the TSLP-DCS system, increase the expression of IL-10 and IFN- $\gamma$, and change the microenvironment of Th2 differentiation to play an anti-allergic effect, and the effect was most obvious when the dose was $4 \mu \mathrm{g} / \mathrm{mL}$. Song Guihua et al.[18] studied the effect of Xiao Qinglong Decoction on the expression of TSLP in airway epithelial cells of asthmatic mice, and the results showed that the expression of TSLP in the bronchus and the expression of TSLP mRNA and TSLP protein in lung tissues of asthmatic mice were significantly increased, while the expression of TSLP was significantly inhibited after the intervention of Xiao Qinglong Decoction. Can effectively control airway allergic inflammation.

\section{Clinical Study}

\subsection{The Acute Phase}

Wang Tongle et al.[19] randomly divided 60 patients with acute stage of chronic obstructive pulmonary disease into treatment group and control group according to the random number table method. The control group received conventional treatment of Western medicine, while the treatment group received conventional treatment of Western medicine and supplemented Xiao Qinglong Decoction. The results indicate that the modified Xiao Qinglong Decoction can improve the clinical symptoms of patients, significantly reduce the c-reactive protein of patients, improve lung function, clinical efficacy is accurate, worthy of clinical promotion. Pan Xiaodan et al.[20] 100 COPD patients with external cold and internal drinking during the acute attack were randomly divided into two groups. The control group received conventional symptomatic support treatment of Western medicine; the observation group was additionally treated with Xiao Qinglong Decoction. After treatment, the scores of individual symptoms and TCM syndrome in the observation group were significantly decreased, and the levels of inflammatory factors (IL-8, IL-4, and TNF- $\alpha$ ) were significantly lower than those in the control group $(\mathrm{P}<0.05)$. Modified Xiao Qinglong Decoction has a definite effect on 
the treatment of lung distension syndrome with external cold and internal drink, which can significantly improve the clinical symptoms of TRADITIONAL Chinese medicine, lung function, and the level of inflammatory factors.

\subsection{Stability}

Professor Zhi[21] took 200 COPD patients at the stable stage as research objects and divided them into the conventional group $(\mathrm{n}=100$, tiotropium treatment) and combined group $(\mathrm{n}=100$, Xiao Qinglong Decoction plus tiotropium treatment) according to the random number table method. After treatment, the results showed that dyspnea index, cough, asthma and sputum score, airway wall thickness, interleukin-6 and C-reactive protein levels in the combined group were significantly lower than those in the conventional group, 6 min walking distance, airway diameter, forced lung capacity, $1 \mathrm{~s}$ forced expiratory volume, CD4+ and CD3+ levels were significantly better than those in the conventional group $(\mathrm{P}<0.05)$. Zhang Chao et al.[22] divided 62 patients with COPD lung qi deficiency and cold syndrome into the reference group and the Traditional Chinese medicine group, with 31 cases in each group. The control group was treated with Western medicine, and the Traditional Chinese medicine group was treated with Xiao Qinglong Decoction and Sanziyangqin Decoction. The results showed that both groups improved after treatment. The total effective rate of the traditional Chinese medicine group was $93.55 \%$ higher than that of the reference group $(77.42 \%)$.

\section{Conclusion}

Chronic obstructive pulmonary disease (COPD) is a common clinical respiratory disease with a long course and poor recovery. Western medicine treatment of COPD, can not fundamentally solve the persistent pulmonary airflow limitation, low lung function, obstructive pulmonary ventilation, airway reconstruction, and other obstacles. TCM adopts treatment based on syndrome differentiation and implements individualized conditioning to enhance individual immunity, improve lung ventilation, relieve hypercoagulability and improve the mental state of the body for COPD. The synergistic effect of TCM and western medicine can be achieved[2,5]. As a common prescription for respiratory system diseases, Xiao Qinglong Decoction has been widely used in the treatment of respiratory system diseases, nervous system, circulation, and other system diseases, and the therapeutic effect is accurate, reflecting the importance of the TCM syndrome differentiation theory system. Modern pharmacology and animal experimental study, and provide the basis for the clinical application of Xiao Qinglong decoction, Xiao Qinglong decoction can be anti-inflammatory, inhibiting reshaping, relieving spasm and improving the signal path, to ensure the effect But at present, the function and mechanism of Xiao Qinglong decoction research are still weak, still need to further study its pharmacology, and mechanism of action, large sample clinical study is less, It is necessary to carry out high-quality research with multi-centers to further explore Xiao Qinglong Decoction, to better serve clinical practice.

\section{References}

[1] Group of Chronic obstructive pulmonary Disease, Respiratory Society of Chinese Medical Association, Working Committee of Chronic obstructive pulmonary Disease, Respiratory Society of Chinese Medical Association. Guidelines for diagnosis and treatment of chronic obstructive pulmonary disease (COPD) $[\mathrm{J}]$. Chin J Tuberculosis Respiratory, 2021, 44(03): 170-205.

[2] Cox NS, McDonald CF, Mahal A, et al. Telerehabilitation for chronic respiratory disease: A randomised controlled equivalence trial[J]. Thorax. 2021, 216934.

[3] Tsai C L, Lan C C, CW Wu, et al. Acupuncture point stimulation treatments combined with Conventional treatment in chronic obstructive pulmonary disease: A systematic review and network meta-analysis[J]. Frontiers in Medicine, 8: 586900.

[4] Zheng Hangbin, Xu Guanglan, Li Guosheng, Li Yuanling, Guo Yihui. Research progress of extracellular vesicles in chronic obstructive pulmonary disease[J]. PLA Medical Journal, 1-11.

[5] Zhang Meicui, Du Shiba, Fan Liang. Clinical efficacy of Xiao Qinglong Decoction combined with routine treatment of acute chronic obstructive pulmonary disease with external cold and internal drinking $[\mathrm{J}]$. Proprietary Chinese Medicine, 2022, 44 (01): 78-82.

[6] Wu Jianjun, Li Xin, Qin Yang, Jiang Liangduo, Jin Ruifeng, Qiu Yue. Application of Xiao Qinglong decoction in chronic obstructive pulmonary disease $[\mathrm{J}]$. Chin J Traditional Chinese Medicine, 2016, 57(22): 1925-1928.

[7] Zhao Y, Chai M S. Protective effect of Asarum and Xiao Qinglong Decoction on respiratory and nervous system injury in cold asthma rats[J/OL]. Chinese Medicinal Materials, 2021 (5): 1232-1236

[8] Ren Tingting, Jin Shoude, Li Zhiheng, Qiang Lixia, Wang Ran, Yang Shanshan.The role of alveolar macrophages in the immune pathogenesis of chronic obstructive pulmonary disease[J]. J Clin Lung, 2019, 24(09): 1724-1728.

[9] Malyshev I, Malyshev Y. Current concept and update of the macrophage plasticity concept: intracellular mechanisms of reprogramming and M3 macrophage "switch" phenotype[J]. Biomed Res Int, 2015, 2015: 341308.

[10] Zheng S. Study on the mechanism of Xiao Qinglong Decoction prescription syndrome based on PI3K/Akt/ mTOR signaling pathway[D]. Shanxi University of Traditional Chinese Medicine, 2021.

[11] Sun Xiaoni. Effect of Bufei Decoction combined with acupoint application on the prevention and treatment of chronic obstructive pulmonary disease syndrome and the effect of inflammation and airway remodeling $[\mathrm{J}]$. Guizhou Pharmaceutical, 2021, 45(06): 910-911.

[12] Wu Nana, Li Meixia, Han Xiaohuan. Effects of Achyranthe bidentata polysaccharide on chronic obstructive pulmonary disease model rats based on TGF- $\beta 1 /$ Smad pathway[J]. Journal of Traditional Chinese Medicine, 2021, 36(09): 1943-1948. 
[13] Ren Yuzhe, Wan Jun, Yu Zhou, Yang Zeyi, Yang Xiangzheng. Effect of Xiao Qinglong Decoction plus Jianjiang Decoction and Erchen Decoction on the treatment of cough variant asthma based on glucocorticoid receptor-mediated TGF- $\beta 1 / \mathrm{Smad} 3$ signal axis $[\mathrm{J}]$. Chinese Journal of Medicine, 2021, 27(08): 10-14.

[14] Peng J P, Chen L R, Hu X C, Cheng X P, Liao L F, Wu L L. Effect of 1,25(OH)_2D_3 on airway inflammation in rats with chronic obstructive pulmonary disease exposed to PM2.5 in vivo[J]. Chinese Journal of Gerontology, 2017, 37(19): 4707-4709.

[15] Yan Wei, Liu Ming, Zhang Qingxiang.Effect of Xiao Qinglong decoction on the level of 1,25 dihydroxyvitamin D_3 in bronchial asthma rats with cold Yin yunfei syndrome[J]. Chinese Journal of Traditional Chinese Medicine, 2017, 35(11): 2806-2809.

[16] Laura K, Zsuzsanna O, Xi C, et al. IL-7Ra Expression Regulates Murine Dendritic Cell Sensitivity to TSLP[J]. Immunol, 2017, 4(12): 3909-3918.

[17] Zhang Lanlan, Yan Juntang, Liu Min, Hu Jinghong, Yu Xue, Fan Fan, Ren Hong. Effects of Xiao Qinglong Decoction on il-4, IL-12, IFN- $\gamma$ and IL-10 expression in DCs induced by TSLP[J]. Chinese Journal of Traditional Chinese Medicine, 2018, 36(06): 1316-1319.

[18] Song Guihua, Zhang Yan, Yu Suping, Lv Weigang, Guan Zhiwei, Mei Xiaofeng, Sun Mengmeng, Wang Jing. Effect of Xiao Qinglong Decoction on TSLP expression in airway epithelial cells of asthmatic model mice[J]. Chongqing Med, 2018, 47(08): 1016-1018 +1022 .

[19] Wang Tongle, Ma Minkai, Wang Suwei, Fan Yanfang, Liu Huirong. Clinical study of Xiao Qinglong Decoction in the treatment of chronic obstructive pulmonary disease in acute phase[J]. Sichuan Traditional Chinese Medicine, 2016, 34(03): 117-119.

[20] Pan Xiaodan, Fan Liang, Lu Baoqiang, Zhang Meicui. Effect of modified Xiao Qinglong Decoction on inflammatory factors in COPD patients with external cold and internal drink during acute attack[J]. Liaoning Journal of Traditional Chinese Medicine, 2018, (09): 1879-1882.

[21] Wang Y, Wang Y, Wang Y, et al. Effects of tiotropium bromide and Xiao Qinglong Decoction on COPD patients in stable stage[J]. Clinical Medical Research and Practice, 2019, 21(8): 119-121.

[22] Zhang Chao, Kang Jianhua, Li Jun. Clinical observation of Xiao Qinglong Decoction and Sanzi Yangqin Decoction in treatment of pulmonary distension (chronic obstructive lung) $[\mathrm{J}]$. Guangming Traditional Chinese Medicine, 2021, 36(17): 2916-2918.

\section{Author Profile}

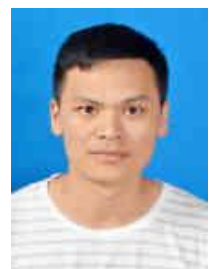

Yun Li, male, postgraduate, main research fields: Prevention and treatment of chronic lung diseases by integrated Traditional Chinese and Western.
Corresponding author: Aiwu Liang, female, (1968-), professor, chief physician, research direction: Prevention and Treatment of Chronic Lung Diseases by Integrated Traditional Chinese and Western Medicine, Ruikang Hospital affiliated to Guangxi University of Chinese Medicine. 